\title{
Supercontinuum Source for Dense Wavelength Division Multiplexing in Square Photonic Crystal Fiber via Fluidic Infiltration Approach
}

\author{
Hamed SAGHAEI \\ Faculty of Engineering, Shahrekord Branch, Islamic Azad University, Shahrekord, Iran \\ h.saghaei@iaushk.ac.ir
}

Submitted March 25, 2016 / Accepted October 30, 2016

\begin{abstract}
In this paper, a square-lattice photonic crystal fiber based on optofluidic infiltration technique is proposed for supercontinuum generation. Using this approach, without nano-scale variation in the geometry of the photonic crystal fiber, ultra-flattened near zero dispersion centered about $1500 \mathrm{~nm}$ will be achieved. By choosing the suitable refractive index of the liquid to infiltrate into the air-holes of the fiber, the supercontinuum will be generated for $50 \mathrm{fs}$ input optical pulse of $1550 \mathrm{~nm}$ central wavelength with $20 \mathrm{~kW}$ peak power. We numerically demonstrate that this approach allows one to obtain more than two-octave spanning of supercontinuum from 800 to $2000 \mathrm{~nm}$. The spectral slicing of this spectrum has also been proposed as a simple way to create multi-wavelength optical sources for dense wavelength division multiplexing.
\end{abstract}

\section{Keywords}

Supercontinuum generation, photonic crystal fiber, optofluidic, dispersion, dense wavelength division multiplexing

\section{Introduction}

Optical supercontinuum (SC) is a coherent and broadband light that is generated when a short laser pulse causes nonlinear effects in nonlinear materials including crystals, glasses, noble gasses and organic liquids [1]. It is unique because of possessing both the bandwidth of a white light source and the coherence properties of a laser $[2,3]$. SC generation naturally requires high intensities which are achieved by confining the energy in space and time [2]. The SC generation mechanism has been recently recognized as a sequence of nonlinear physical processes, each occurring consecutively along the propagation axis such as self-phase modulation (SPM), self-steepening (SS), stimulated Raman scattering (SRS), and four-wave mixing (FWM) [4]. The first SC generation experiments in silica were obtained by standard optical fiber with zero group velocity dispersion (GVD) wavelength around $1.3 \mu \mathrm{m}$ and several hundred meters of the fiber length [5]. The advent of photonic crystal fibers (PCFs) in the form of triangle and square lattices at the late 1990s attracted widespread interest throughout the scientific community, and has led to a revolution in the generation of ultra-broadband high brightness spectra through SC generation [6-8]. The single-mode propagation over broad wavelength ranges, the enhanced modal confinement, the elevated nonlinearity, and the ability to engineer group velocity dispersion are the unique and excellent characteristics of PCFs [8]. Low dispersion is the key parameter for spectral broadening and other nonlinear phenomena such as FWM [9]. It enables single-mode phase matching of the nonlinear processes that broaden the spectrum. Optimizing dispersion relaxes the need for high intensity, allowing SC generation for larger cores, longer pulses and shorter length of the fiber [10]. It should be noted that the reduced effective area of the propagating mode in PCF enhances the Kerr nonlinearity effect relative to standard fiber [11]. SC generation in PCFs has many applications in optical frequency metrology [12], optical coherence tomography (OCT) [13], pulse compression [14], and the design of tunable ultrafast femtosecond laser sources [15]. In a telecommunication context, the spectral slicing of broadband SC spectra has also been proposed as a simple way to create multi-wavelength optical sources for dense wavelength division multiplexing (DWDM) applications [16, 17]. There are various techniques that the dispersion of PCF can be engineered and customized for a particular application. However, most of the proposed techniques, so far, are based on varying the PCF geometry; such as varying the circular air-hole diameter $(d)[18,19]$, the pitch size $(\Lambda)$ of the periodic lattice [9], as well as the number of air-holes rings $(N)$ surrounding the PCF core or even using ring-shaped air-holes [20]. These techniques depend on the technological capability to realize a specific design with high precision, and also are limited by the size of the PCF cross-sectional area. In particular, it is quite difficult to control the accurate positions and radii of the air-holes within the PCF both triangle and square lattices. Precision in control of these two geometrical parameters during the fabrication process in addition to the maximum number of the rings of air-holes within 
a PCF are the most critical constraints in acquiring the desired dispersion. To overcome these topological limitations, optofluid approach is proposed and our focus in this paper for dispersion engineering is based on the optofluidic: A new branch in photonic that attempts to merge fluidic and optic [21]. In a meticulous manner, due to their intrinsic porous nature photonic crystal $(\mathrm{PhC})$ devices infiltrated with optofluid have demonstrated tunable and reconfigurable optical properties [22]. Furthermore, selective liquid infiltration of individual air pores of a planar $\mathrm{PhC}$ lattice has shown to extend the number of opportunities associated with this optofluidic platform [23-25]. This offers the potential for realizing integrated microphotonic devices and circuits, which could be (re)configured by simply changing the liquid and/or the pattern of the infiltrated area within the $\mathrm{PhC}$ lattice.

Our research group numerically investigated the potential of selective optofluidic infiltration of air-holes within the triangular-lattice photonic crystal fiber (TPCF) in [24] in which different properties of TPCF composed of silica background were studied. The aim of that study was to investigate the effects of fluid infiltration on the dispersion profile and its nonlinear parameter to obtain optimal structure of the PCF at the desired zero dispersion wavelength depending on the application. In this paper, a square-lattice photonic crystal fiber (SPCF) based on optofluidic infiltration technique is proposed for supercontinuum (SC) generation. Using this approach, without nano-scale variation in the geometry of the SPCF, ultraflattened near zero dispersion centered about $1500 \mathrm{~nm}$ will be achieved. By choosing the suitable refractive index of the liquid to infiltrate into the air-holes of the SPCF, the $\mathrm{SC}$ will be generated for $50 \mathrm{fs}$ input optical pulse of $1550 \mathrm{~nm}$ central wavelength with $20 \mathrm{~kW}$ peak power. We numerically demonstrate that this approach allows one to obtain more than two-octave spanning of SC from 800 to $2000 \mathrm{~nm}$. The spectral slicing of this spectrum has also been proposed as a simple way to create multi-wavelength optical sources for dense wavelength division multiplexing.

The rest of this paper is organized as follows. In Sec. 2, we propose the procedure for dispersion engineering of solid core SPCF, by means of selective optofluidic infiltration. The mathematical analysis of SC generation is presented in Sec. 3. Section 4 is dedicated to the discussion of the simulation results. Finally, we will conclude the paper in Sec. 5.

\section{Dispersion Engineering of SPCF}

The goal of this section is to investigate the possibility of tailoring the dispersion profile of a SPCF by means of selective optofluidic infiltration of SPCF's airholes to achieve SC generation. In doing so, we have we have considered a solid core SPCF that consists of circular air-holes of diameter $d=0.6 \Lambda$ arranged in a square-lattice of constant $\Lambda=2 \mu \mathrm{m}$, as depicted in Fig. 1(a).
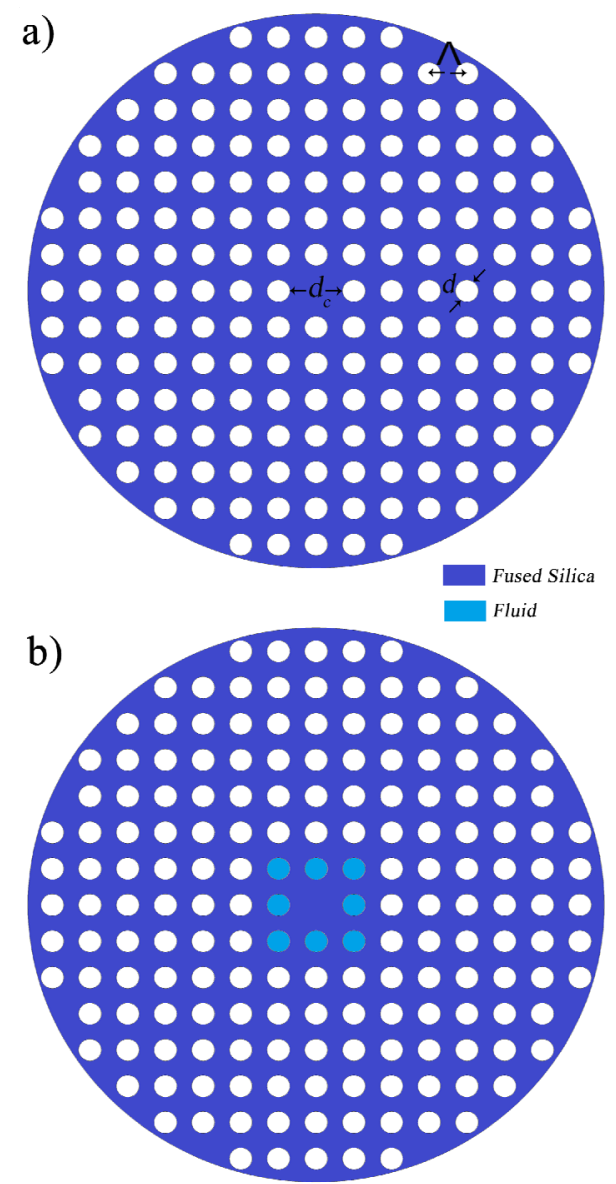

Fig. 1. A cross-sectional view of (a) un-infiltrated SPCF and (b) the inner most ring of SPCF to be selectively infiltrated with an optofluidic of index $n_{\mathrm{f}}$, two have the same the lattice constant of $2 \mu \mathrm{m}$ and air-hole diameters of $0.6 \Lambda$.

The air-holes lattice forms seven square rings co-centered with the solid core. Figure 1(b) illustrates the case for which the air-holes form the inner most ring which is infiltrated by an optofluidic of refractive index $n_{\mathrm{f}}$. The white and dark circles in Fig. 1 represent the un-infiltrated and selectively infiltrated air-holes, respectively. In order to investigate the dispersion properties of the SPCF, we have employed a full vectorial finite element method (FV-FEM) [26]. The refractive index of silica as a background material can be expressed by the Sellmeier expansion as

$$
n(\lambda)=1+\sum_{n=1}^{3} \frac{A_{n} \lambda^{2}}{\lambda^{2}-B_{n}^{2}}
$$

where $A_{n}$ and $B n$ are the Sellmeier coefficients. For silica, their values are as follows [27]: $A_{1}=0.6961663$, $A_{2}=0.407942, \quad A_{3}=0.897479, \quad B_{1}=0.068404 \mu \mathrm{m}^{2}$, $B_{2}=0.116241 \mu \mathrm{m}^{2}$, and $B_{3}=9.89616 \mu \mathrm{m}^{2}$.

Eigenvalues and the propagation constants $k_{\mathrm{z}}$ are obtained by solving the previous equations. Effective refractive index $n_{\text {eff }}$ is calculated as

$$
n_{\mathrm{eff}}=\frac{k_{z}}{k_{0}} .
$$




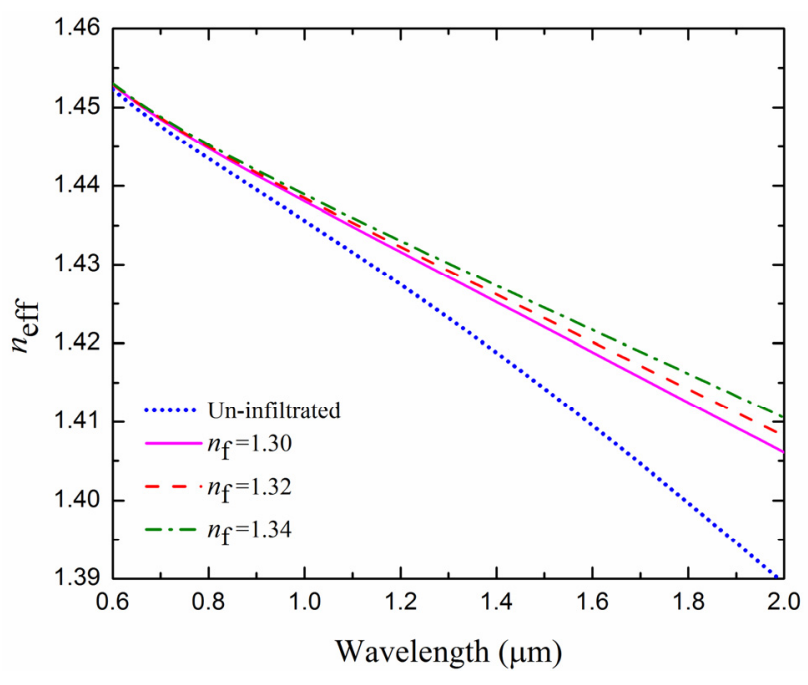

Fig. 2. Comparison of the effective refractive index for the un-infiltrated SPCF of Fig. 1(a) with Fig. 1(b) infiltrated with optical fluids of various indices $\left(1.3 \leq n_{\mathrm{f}} \leq 1.34\right)$.

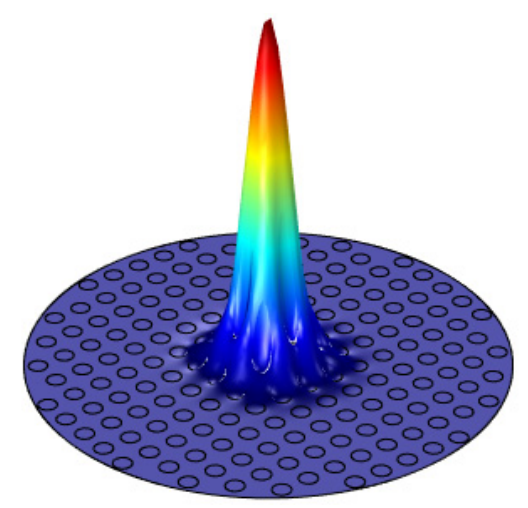

Fig. 3. 3D schematic view of fundamental mode distribution for the SPCF with the inner most ring to be selectively infiltrated with an optofluidic for $n_{\mathrm{f}}=1.30$.

Figure 2 compares effective refractive index for the un-infiltrated SPCF of Fig. 1(a) with Fig. 1(b) infiltrated with optical fluids such as water, methanol, and ethanol with refractive indices of $1.3,1.32$ and 1.34 in $1550 \mathrm{~nm}$, respectively. The refractive indices versus wavelength of methanol and ethanol are given by:

$$
n(\lambda)= \begin{cases}\sqrt{1.74-0.005 \lambda^{2}+0.004 \lambda^{-2}}, & \text { methanol } \\ \sqrt{1+\frac{0.83 \lambda^{2}}{\lambda^{2}-0.009}-\frac{0.156 \lambda^{2}}{\lambda^{2}-0.494}}, & \text { ethanol }\end{cases}
$$

In the following simulations, the dependencies of refractive indices of fluids on wavelength are taken into account. The total dispersion $D$ is computed as

$$
D=\left(\frac{-\lambda}{c}\right) \frac{\mathrm{d}^{2} n_{\text {eff }}}{\mathrm{d} \lambda^{2}} .
$$

The 3D schematic view of the fundamental mode distribution is shown in Fig. 3 for the case of $n_{\mathrm{f}}=1.32$. Fundamental mode assumption is used for the rest of the paper.

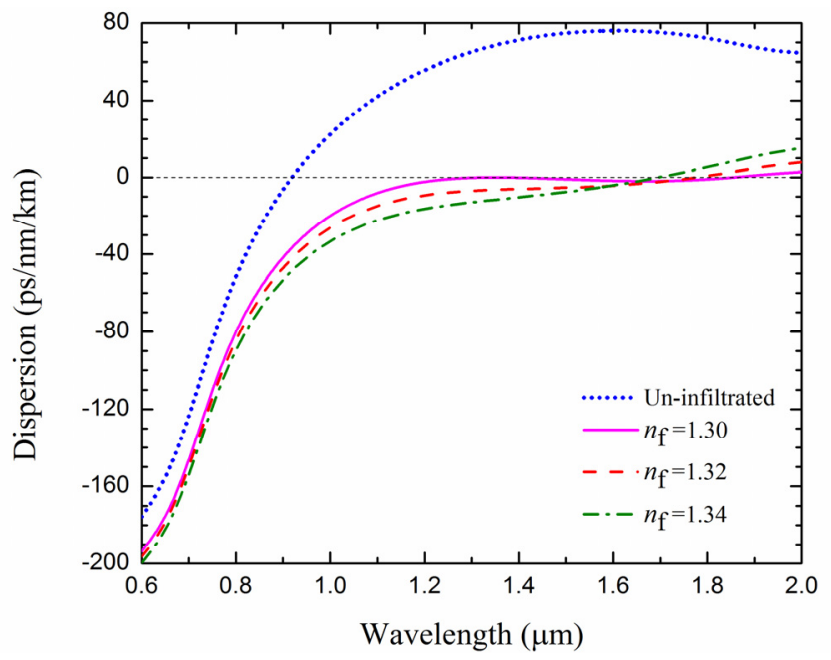

Fig. 4. Comparison of the dispersion for the un-infiltrated SPCF of Fig. 1(a) with those of Fig. 1(b) infiltrated with optical fluids of various indices $\left(1.3 \leq n_{\mathrm{f}} \leq 1.34\right)$.

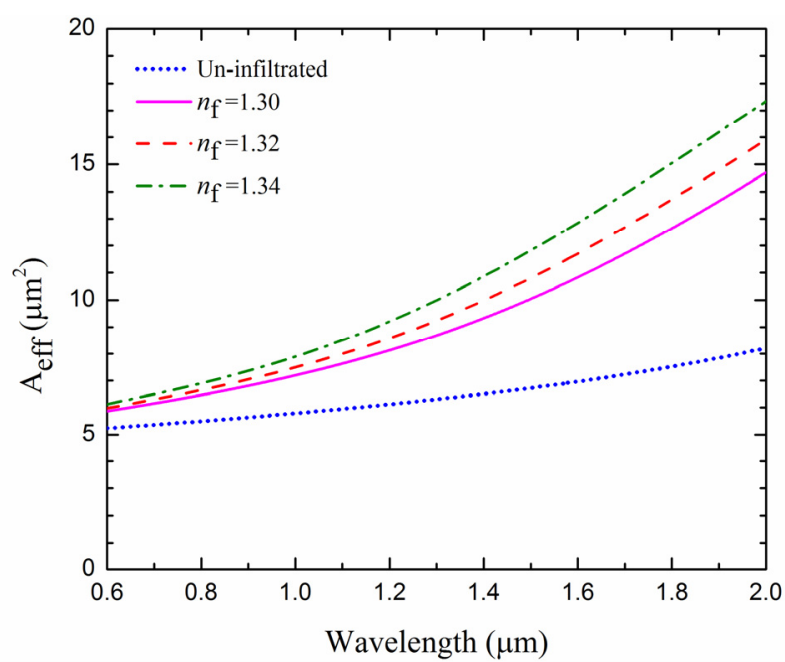

Fig. 5. Comparison of effective mode for the un-infiltrated SPCF of Fig. 1(a) with Fig. 1(b) infiltrated with optical fluids of various indices $\left(1.3 \leq n_{\mathrm{f}} \leq 1.34\right)$.

In order to investigate the effect of the infiltration on the SPCF dispersion and effective mode area profiles, we compare these profiles for the un-infiltrated and infiltrated cases. Figures 4 and 5 compare the numerical results of dispersion profiles and effective mode area for $1.3 \leq n_{\mathrm{f}} \leq 1.34$, respectively. The comparison shown in Fig. 4 reveals that infiltrating the most inner ring of the SPCF with various optical fluids reduces the SPCF dispersion value significantly and also enables one to design SPCFs suitable for various applications such as dispersion flattened PCF (DF-PCF), dispersion shifted PCF (DSPCF), and dispersion compensated PCF (DC-PCF). For example, this figure shows that the infiltration with $n_{\mathrm{f}}=1.3$ has reduced the dispersion profile, in the wavelength range of $1.2 \mu \mathrm{m}<\lambda<2 \mu \mathrm{m}$, to insignificant values of $0 \leq D \leq 2$ (ps/km.nm). This nearly flattened profile is an example of DF-PCF, over the given range of the wavelengths that makes this particular infiltrated PCF suitable for DWDM applications that can be used in the optical telecommunication systems. 
Furthermore, the comparison of curves of Fig. 5 illustrates that by increasing the value of refractive index infiltration, the effective mode area increases and consequently the value of nonlinear coefficient, $\gamma$ decreases as

$$
\gamma=\frac{n_{2} \omega_{0}}{c A_{\text {eff }}\left(\omega_{0}\right)}
$$

where $n_{2}$ is the nonlinear index which is assumed by $2.7 \times 10^{-20} \mathrm{~m}^{2} / \mathrm{W}$ for silica. $c$ is the speed of light. Results show that there is a trade-off between the dispersion and nonlinear coefficient by infiltrating the fluid. For this reason, we restrict our simulations only to the case of most inner ring infiltration.

\section{Mathematical Background}

Nonlinear Schrödinger equation (NLSE) can truly simulate the pulse propagation in single mode SPCF and can be derived under the slowly varying envelope approximation as [4]

$$
\begin{aligned}
& \frac{\partial A}{\partial z}+\frac{\alpha}{2} A+\sum_{n=1}^{4} i^{(n-1)} \frac{\beta_{n}}{n !} \frac{\partial^{n} A}{\partial t^{n}}= \\
& \quad i\left(\gamma\left(\omega_{0}\right)+i \gamma_{1} \frac{\partial}{\partial t}\right) \cdot A(z, t) \int_{-\infty}^{t} R\left(t^{\prime}\right)\left|A\left(z, t-t^{\prime}\right)\right|^{2} \mathrm{~d} t
\end{aligned}
$$

where $A(z, t)$ is the intensity temporal profile of the pulse, $\alpha$ is the fiber loss, $\beta$-terms correspond to the chromatic dispersion of the fiber. The mode propagation constant is:

$$
\begin{aligned}
& \beta(\omega)=n(\omega) \frac{\omega}{c}=\sum \frac{\beta_{m}}{m !}\left(\omega-\omega_{0}\right)^{m}, \\
& \beta_{m}=\left(\frac{\mathrm{d}^{m} \beta}{\mathrm{d} \omega^{m}}\right)_{\omega=\omega_{0}}
\end{aligned}
$$

where $\beta_{m}$ describes the wave-vector of the light in the fiber. The term proportional to $\beta_{2}$ is responsible for group velocity dispersion (GVD). It causes temporal pulse broadening when a pulse propagates in a single-mode fiber. Higher orders of dispersion become dominant when the input pulse central wavelength is near the zero dispersion wavelength or when the bandwidth of the pulse becomes a significant fraction of the central frequency. Figure 6 compares dispersion characteristics of the un-infiltrated SPCF of Fig. 1(a) with those of Fig. 1(b). The nonlinear parameter $\gamma$ is a very important parameter that determines the magnitude of the optical nonlinearity and leads to SPM, self-steepening (SS), stimulated Raman scattering (SRS), and FWM. The right-hand side of (6) accounts for the nonlinear response of the fiber. The response function $R(t)$ including instantaneous Kerr and delayed Raman response effects can be written as

$$
R(t)=\left(1-f_{\mathrm{R}}\right) \delta(t)+f_{\mathrm{R}} h_{\mathrm{R}}(t)
$$

where $f_{\mathrm{R}}=0.18$ represents the fractional contribution of the delayed Raman response. The Raman response function $h_{\mathrm{R}}(\mathrm{t})$ takes an approximate analytic form as $\left(\tau_{1}=0.12 \mathrm{fs}\right.$ and $\tau_{2}=0.32$ fs for silica)

$$
h_{\mathrm{R}}(t)=\frac{\tau_{1}^{2}+\tau_{2}^{2}}{\tau_{1} \tau_{2}} \exp \left(-t / \tau_{2}\right) \sin \left(t / \tau_{1}\right)
$$
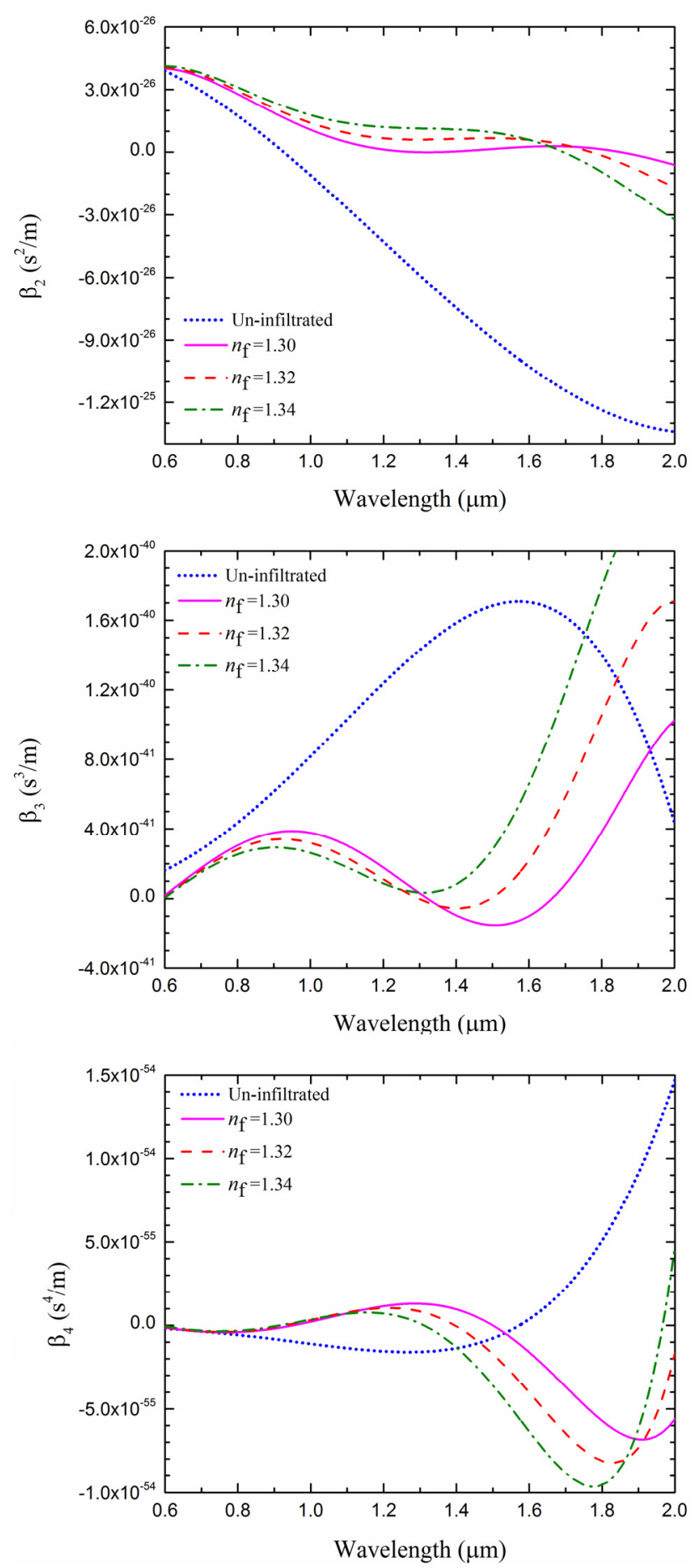

Fig. 6. Cross-sectional view of (a) un-infiltrated SPCF and (b) the inner most ring of SPCF to be selectively infiltrated with an optofluidic of index $n_{\mathrm{f}}$, two have the same the lattice pitch $(\Lambda=2 \mu \mathrm{m})$ and air-hole diameters $(d=0.6 \Lambda)$. 
The Split-Step Fourier Method (SSFM) is used to solve the NLSE which has proven to be a well-suited numerical technique. The input pulse is given by

$$
A(0, T)=\sqrt{P_{0}} \operatorname{sech}\left(\frac{T}{T_{0}}\right) .
$$

At $z=0$, and define soliton order $N$ is written as

$$
N^{2}=\frac{T_{0}^{2} \gamma P_{0}}{\left|\beta_{2}\right|} .
$$

The soliton order $N$ is determined by the characteristics of input pulse $\left(T_{0}\right.$ and $\left.P_{0}\right)$, and the properties of the fiber $\left(\gamma\right.$ and $\left.\beta_{2}\right)$. In the mathematically ideal case, where $A$ is a perfectly hyperbolic-secant-shaped pulse, $N$ is an integer, and only self-phase modulation and anomalous group velocity dispersion are present, higher-order solitons propagate in a periodic evolution pattern with original shape recurring at multiples of the soliton period. This is called as ideal soliton conditions. Without any perturbation, these higher order solitons will propagate in a periodic evolution pattern and SC generation would never occur. SC generation is the result of deviations from ideal soliton conditions experienced by a higher order soliton. It means that a small perturbation results in a higher order soliton with the order of $N$ breaks up to $N$ fundamental solitons [25, 28, 29].

These small perturbations include higher order dispersion, SS and SRS. As a result of self-frequency shift (SFS) induced by intrapulse Raman scattering, the fundamental solitons continuously shift toward the longer wavelength of the broadened spectrum, causing a considerable spectral expansion on the red side. The blue side of the spectrum is developed due to the blue shifted nonsolitonic radiation (NSR) [28] or phase-matched. The SPM leads to initially symmetrical spectral broadening of optical pulses after which the phase matching conditions are fulfilled. Then soliton fission occurs and finally SFS and NSR shift the spectral components toward longer and shorter wavelength, respectively and SC will be generated.

\section{Simulation Results}

Section 2 gives us a qualitative idea about the engineering of the dispersion which is suitable for SC generation in SPCF, now, it is crucial to use the extracted parameters of the presented figures to take into account the influence of dispersion and fluid injection on SC generation. We calculate the $\mathrm{SC}$ generation using the model of (6), for input source of $50 \mathrm{fs}$ pulse duration, $20 \mathrm{~kW}$ peak power $(50 \mathrm{~mW}$ average power) and the center wavelength of $1550 \mathrm{~nm}$. In this wavelength different commercial Er-doped femtosecond fiber lasers, such as Femtolite Ultra Bx-60, the CF1550-HP, and Buccaneer are available [30]. The simulations are run for the $100-\mathrm{mm}$ long engineered SPCF with the dispersion and effective mode area calculated in Figs. 4, and 5, respectively. Figure 7 shows spectrum evaluation of SC generation for
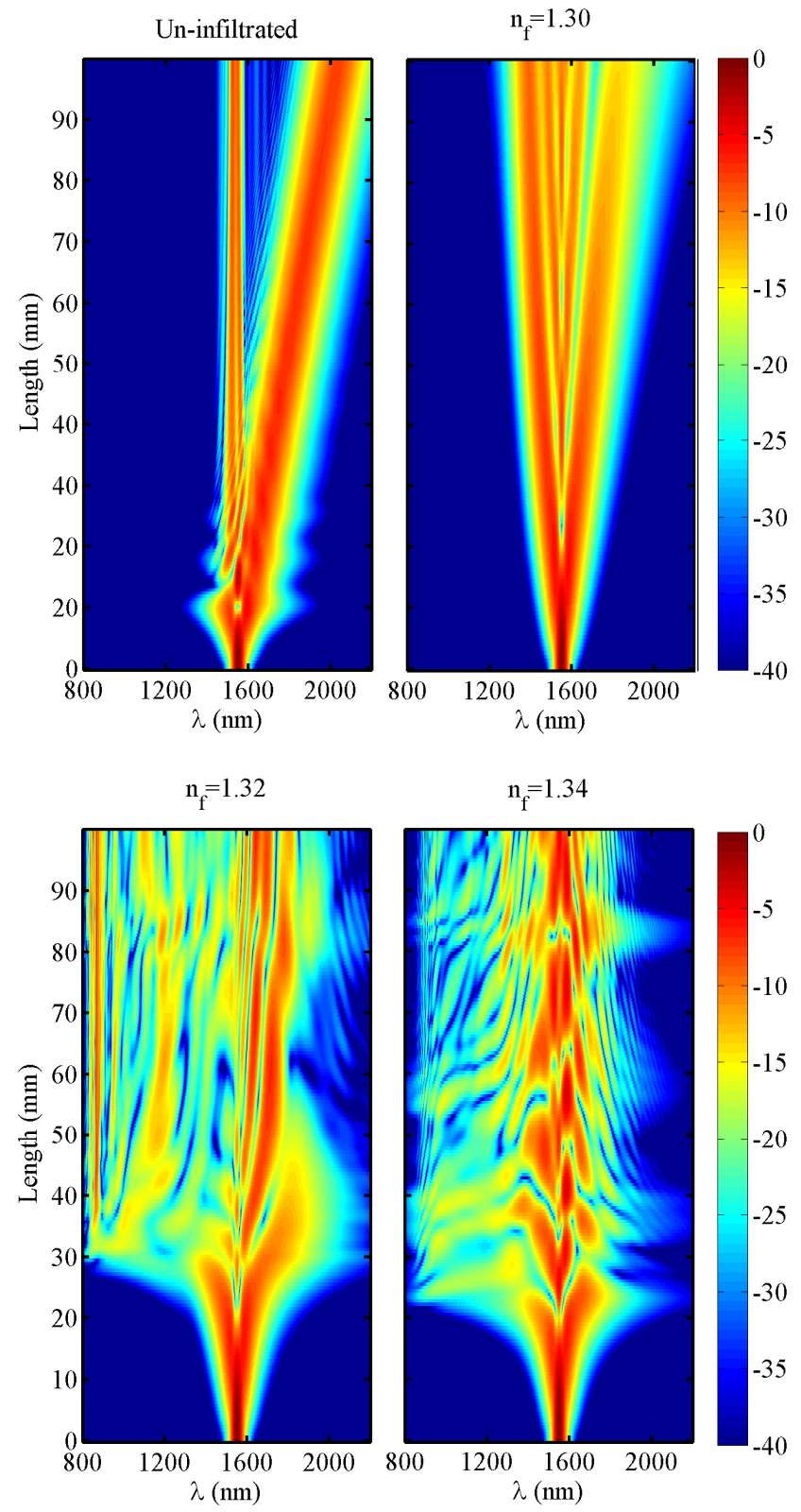

Fig. 7. Simulated evolution of the SC spectrum over propagation distance through SPCF for the uninfiltrated case, $1.3,1.32$, and 1.34 the infiltration case when pumped at $1550 \mathrm{~nm}$.

infiltrations with $1.3 \leq n_{\mathrm{f}} \leq 1.34$ (shown in Fig. 1(b)) with the uninfiltrated case when setting the pump wavelength in the $1550 \mathrm{~nm}$. The simulation results reveal that we can obtain an optimum SC generation simply by changing the fluid refractive index through the SPCF holes. For input pulse with a central wavelength corresponding to $1550 \mathrm{~nm}$, the suitable fluid has a refractive index of 1.32 to have ultra-broadband SC as wide as $1200 \mathrm{~nm}$ from 800 to $2000 \mathrm{~nm}$.

Aiming to study the coherence degradation, the analysis of the coherence properties of the generated supercontinua from the presented PCF infiltrated by fluids is performed. It considers the addition of one photon per mode noise with a random phase and it is evaluated within the first order degree of coherence given by 


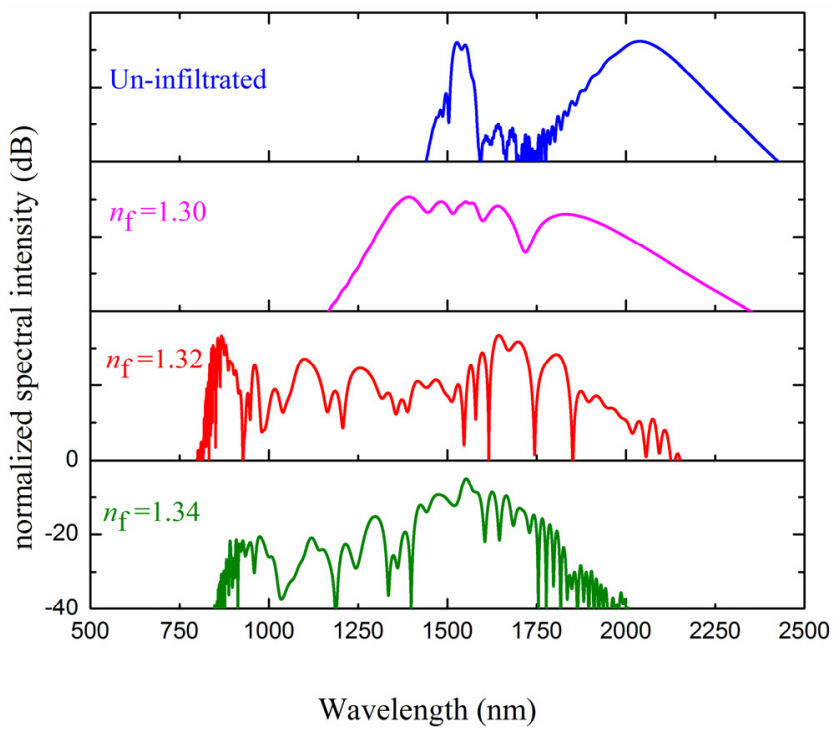

Fig. 8. SC coherence at the output of the fiber for the uninfiltrated case, $1.30,1.32$, and 1.34 the infiltration case when pumped at $1550 \mathrm{~nm}$.

$$
\left|g_{12}^{(1)}\left(\lambda, t_{1}-t_{2}=0\right)\right|=\frac{\left|\left\langle E_{1}^{*}\left(\lambda, t_{1}\right) E_{2}\left(\lambda, t_{2}\right)\right\rangle\right|}{\sqrt{\left\langle\left|E\left(\lambda, t_{1}\right)\right|^{2}\right\rangle\left\langle\left|E\left(\lambda, t_{1}\right)\right|^{2}\right\rangle}}
$$

where $E_{1}(\lambda)$ and $E_{2}(\lambda)$ are two electric fields. Angular brackets indicate an ensemble average over independently generated pairs of SC spectra, and $t$ is the time measured at the scale of the temporal resolution of the spectrometer used to resolve these spectra. $g_{12}$ is considered at $t_{1}-t_{2}=0$ in order to focus on the wavelength dependence of the coherence. It is known that the coherence property of SC light is greatly affected by the quantum noise of the pump pulse. From an ensemble average of 50 independent simulations, we find that the generated supercontinuum in the $100 \mathrm{~mm}$ long PCF infiltrated by methanol with an input peak power of $20 \mathrm{~kW}$ is coherent over the entire generated bandwidth that is shown in Fig. 8 in which methanol has the flattest coherence among them. It shows that SPCF infiltrated by the fluid of $n_{\mathrm{f}}=1.32$ is a suitable source for dense wavelength division multiplexing systems. Extracting the desired bandwidth of high intensity and coherence optical signal can be done using some optical filters such as optical fiber Bragg grating that is constructed in a short segment of optical fiber [31].

\section{Conclusion}

In summary, we investigated and discussed some nonlinear phenomena that result in SC generation in SPCF and proposed optofluidic infilteration as an alternative approach. We numerically investigated the generation of $\mathrm{SC}$ with and without optofluidic infilteration and our results confirmed that using the proposed approach, without nano-scale variation in the geometry of the SPCF, its dispersion was simply engineered as desired for fluid with $n_{\mathrm{f}}$ $=1.32$. Also, for $1550 \mathrm{~nm}$ central wavelength of an optical pulse with 50 fs duration and $20 \mathrm{~kW}$ peak power, SC with $1200 \mathrm{~nm}$ spectral spanning was generated in $100-\mathrm{mm}$ length of the fiber. The generated SC is a suitable source for wavelength division multiplexing system.

\section{References}

[1] DUDLEY, J. M., COEN, S. Coherence properties of supercontinuum spectra generated in photonic crystal and tapered optical fibers. Optics Letters, 2002, vol. 27, no. 13, p. 1180-1182. ISSN: 1539-4794. DOI: 10.1364/OL.27.001180

[2] DUDLEY, J. M., GENTY, G., COEN, S. Supercontinuum generation in photonic crystal fiber. Reviews of Modern Physics, 2006, vol. 78 , no. 4 , p. 1135-1184. ISSN: 0034-6861. DOI: 10.1103/RevModPhys.78.1135

[3] DUDLEY, J. M., TAYLOR, J. R. Supercontinuum Generation in Optical Fibers. Cambridge University Press, 2010. ISBN: 1139486187

[4] AGRAWAL, G. P. Nonlinear Fiber Optics. Academic Press, 2007. ISBN: 0123695163

[5] BIRKS, T., BAHLOUL, D., MAN, T., et al. Supercontinuum generation in tapered fibres. In Proceedings of Lasers and ElectroOptics, CLEO'02. USA, 2002, p. 486-487. isbn: 1557527067. DOI: $10.1109 /$ CLEO.2002.1034235

[6] RANKA, J. K., WINDELER, R. S., STENTZ, A. J. Visible continuum generation in air-silica microstructure optical fibers with anomalous dispersion at $800 \mathrm{~nm}$. Optics Letters, 2000, vol. 25 , no. 1, p. 25-27. ISSN: 1539-4794. DOI: $10.1364 /$ OL. 25.000025

[7] WADSWORTH, W. J., ORTIGOSA-BLANCH, A., KNIGHT, J. C., et al. Supercontinuum generation in photonic crystal fibers and optical fiber tapers: a novel light source. Journal of the Optical Society of America B, 2002, vol. 19, no. 9, p. 2148-2155. ISSN: 1520-8540. DOI: $10.1364 / J O S A B .19 .002148$

[8] REEVES, W., SKRYABIN, D. V., BIANCALANA, F., et al. Transformation and control of ultra-short pulses in dispersionengineered photonic crystal fibres. Nature, 2003, vol. 424, no. 6948, p. 511-515. ISSN: 0028-0836. DOI: 10.1038 /nature 01798

[9] MONAT, C., EBNALI-HEIDARI, M., GRILLET, C., et al. Fourwave mixing in slow light engineered silicon photonic crystal waveguides. Optics Express, 2010, vol. 18, no. 22, p. 22915 to 22927. ISSN: 1094-4087. DOI: 10.1364/OE.18.022915

[10] SHEN, L., HUANG, W.-P., CHEN, G., et al. Design and optimization of photonic crystal fibers for broad-band dispersion compensation. IEEE Photonics Technology Letters, 2003, vol. 15, no. 4 , p. 540-542. ISSN: 1041-1135. DOI: 10.1109/LPT.2003.809322

[11] MORTENSEN, N. A. Effective area of photonic crystal fibers. Optics Express, 2002, vol. 10, no. 7, p. 341-348. ISSN: 10944087. DOI: $10.1364 /$ OE. 10.000341

[12] UDEM, T., HOLZWARTH, R., HÄNSCH, T. W. Optical frequency metrology. Nature, 2002 , vol. 416 , no. 6877 , p. 233 to 237. ISSN: 0028-0836. DOI: $10.1038 / 416233 \mathrm{a}$

[13] MOON, S., KIM, D. Y. Ultra-high-speed optical coherence tomography with a stretched pulse supercontinuum source. Optics Express, 2006, vol. 14, no. 24, p. 11575-11584. ISSN: 1094-4087. DOI: $10.1364 / \mathrm{OE} .14 .011575$

[14] SCHENKEL, B., PASCHOTTA, R., KELLER, U. Pulse compression with supercontinuum generation in microstructure 
fibers. Journal of the Optical Society of America B, 2005, vol. 22, no. 3 , p. 687-693. ISSN: 1520-8540. DOI: 10.1364/JOSAB.22.000687

[15] LIU, B., HU, M., FANG, X., et al. High-power wavelength-tunable photonic-crystal-fiber-based oscillator-amplifier-frequencyshifter femtosecond laser system and its applications for material microprocessing. Laser Physics Letters, 2008, vol. 6, no. 1, p. 44. ISSN: 1612-202X. DOI: 10.1002/lapl.200810084

[16] NAKASYOTANI, T., TODA, H., KURI, T., et al. Wavelengthdivision-multiplexed millimeter-waveband radio-on-fiber system using a supercontinuum light source. Journal of Lightwave Technology, 2006, vol. 24, no. 1, p. 404-410. ISSN: 0733-8724. DOI: $10.1109 /$ JLT.2005.859854

[17] SAGHAEI, H., SEYFE, B., BAKHSHI, H., et al. Novel approach to adjust the step size for closed-loop power control in wireless cellular code division multiple access systems under flat fading. IET Communications, 2011, vol. 5, no. 11, p. 1469-1483. ISSN: 1751-8636. DOI: 10.1049 /iet-com.2010.0029

[18] HANSEN, K. P., FOLKENBERG, J. R., PEUCHERET, C., et al. Fully dispersion controlled triangular-core nonlinear photonic crystal fiber. In Proceedings of Optical Fiber Communication Conference. Atlanta (GA, USA), 2003, p. 505-509. ISBN: 155752-731-8.

[19] WU, T.-L., CHAO, C.-H. A novel ultra flattened dispersion photonic crystal fiber. IEEE Photonics Technology Letters, 2005, vol. 17, no. 1, p. 67-69. ISSN: 1041-1135. DOI: 10.1109/LPT.2004.837475

[20] SAGHAEI, H., HEIDARI, V., EBNALI-HEIDARI, M., et al. A systematic study of linear and nonlinear properties of photonic crystal fibers. Optik-International Journal for Light and Electron Optics, 2015, vol. 127, no. 24, p. 11938-11947. ISSN: 0030-4026. DOI: $10.1016 /$ j.jileo.2016.09.111

[21] MONAT, C., DOMACHUK, P., EGGLETON, B. Integrated optofluidics: A new river of light. Nature Photonics, 2007, vol. 1, no. 2 p. 106-114. ISSN: 1749-4885. DOI: 10.1038/nphoton.2006.96

[22] MONAT, C., DOMACHUK, P., GRILLET, C., et al. Optofluidics: a novel generation of reconfigurable and adaptive compact architectures. Microfluidics and Nanofluidics, 2008, vol. 4, no. 12, p. 81-95. ISSN: 1613-4982. DOI: 10.1007/s10404-007-0222-z

[23] EBNALI-HEIDARI, M., DEHGHAN, F., SAGHAEI, H., et al. Dispersion engineering of photonic crystal fibers by means of fluidic infiltration. Journal of Modern Optics, 2012, vol. 59, no. 16, p. 1384-1390. ISSN: 0950-0340. DOI: $10.1080 / 09500340.2012 .715690$

[24] EBNALI-HEIDARI, M., SAGHAEI, H., KOOHI-KAMALI, F., et al. Proposal for supercontinuum generation by optofluidic infiltrated photonic crystal fibers. IEEE Journal of Selected Topics in Quantum Electronics, 2014, vol. 20, no. 5, p. 582-589. ISSN: 1077-260X. DOI: 10.1109/JSTQE.2014.2307313

[25] SAGHAEI, H., EBNALI-HEIDARI, M., MORAVVEJ-FARSHI, M. K. Midinfrared supercontinuum generation via $\mathrm{As}_{2} \mathrm{Se}_{3}$ chalcogenide photonic crystal fibers. Applied Optics, 2015, vol. 54, no. 8, p. 2072-2079. ISSN: 1539-4522. DOI: 10.1364/AO.54.002072

[26] SAITOH, K., KOSHIBA, M. Full-vectorial finite element beam propagation method with perfectly matched layers for anisotropic optical waveguides. Journal of Lightwave Technology, 2001, vol. 19 , no. 3, p. 405. ISSN: 0733-8724. DOI: 10.1109/50.918895

[27] MALITSON, I. Interspecimen comparison of the refractive index of fused silica. Journal of the Optical Society of America, 1965, vol. 55, no. 10 , p. 1205-1208. ISSN: 0030-3941. DOI: 10.1364/JOSA.55.001205

[28] DIOUF, M., SALEM. A., CHERIF, R., et al. Super-flat coherent supercontinuum source in $\mathrm{As}_{38.8} \quad \mathrm{Se}_{61.2}$ chalcogenide photonic crystal fiber with all-normal dispersion engineering at a very low input energy. Applied Optics, 2017, vol. 56, no. 2, p. 163-169. ISSN: 1539-4522. DOI: 10.1364/AO.56.000163

[29] SAgHAEI, H., MORAVVEJ-FARSHI, M. K., EBNALIHEIDARI, M., et al. Ultra-wide mid-infrared supercontinuum generation in $\mathrm{As}_{40} \mathrm{Se}_{60}$ chalcogenide fibers: Solid core PCF versus SIF. IEEE Journal of Selected Topics in Quantum Electronics, 2016, vol. 22, no. 2, p. 1-8. ISSN: 1077-260X. DOI: 10.1109/JSTQE.2015.2477048

[30] MIRET, J., SILVESTRE, E., ANDRÚS, P. Octave-spanning ultraflat supercontinuum with soft-glass photonic crystal fibers. Optics Express, 2009, vol. 17, no. 11, p. 9197-9203. ISSN: 10944087. DOI: 10.1364/OE.17.009197

[31] KASHYAP, R. Fiber Bragg Gratings. Academic Press, 1999. ISBN: 0080506275

\section{About the Author ...}

Hamed SAGHAEI was born in Shahrekord, Iran, in 1982. $\mathrm{He}$ received the B.Sc., M.Sc. and Ph.D. degrees from Amirkabir University of Technology, Shahed University, and Science and Research branch of IAU, Tehran, Iran, all in Electrical Engineering in 2004, 2007, and 2015, respectively. He joined Islamic Azad University, Shahrekord, Iran, in 2011, where he is currently an assistant professor of electronics and the head of the Faculty of Electrical and Electronic Engineering. His research interests are photonic crystal fibers and waveguides, supercontinuum generation, and optical communication. 